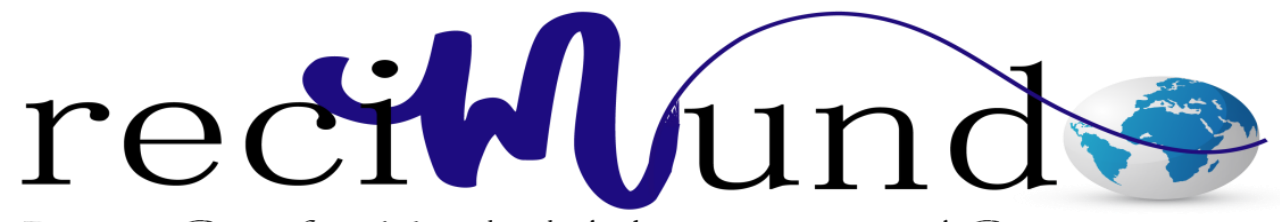

Revista Científica Mundo de la Investigación y el Conocimiento

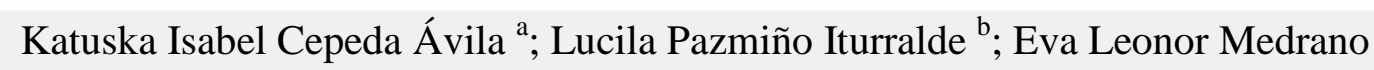
Freire $^{\mathrm{c}}$

Evolución de la Investigación Científica en América Latina

Revista Científica Mundo de la Investigación y el Conocimiento. Vol. 2 núm.2, mayo, ISSN: 2588-073X, 2018, pp. 464-476

DOI: 10.26820/recimundo/2.(2).2018.464-476

Editorial Saberes del Conocimiento

Recibido: 05/12/2017

Aceptado: 25/03/2018

a. Universidad de Guayaquil; katuska.cepedaa@ug.edu.ec

b. Universidad de Guayaquil; $\underline{\text { ucil.pazminoi@ug.edu.ec }}$

c. Universidad de Guayaquil; eva.medranof@ug.edu.ec 


\section{Evolución de la Investigación Científica en América Latina}

Vol. 2, núm. 2., (2018)

Katuska Isabel Cepeda Ávila; Lucila Pazmiño Iturralde; Eva Leonor Medrano Freire

\section{RESUMEN}

El presente trabajo sintetiza la evolución en la investigación científica en América Latina a través de los años y todo lo referente al crecimiento en la producción de la investigación en ciencia y tecnología de cara a las publicaciones científicas a nivel internacional. El desarrollo de la investigación científica por parte de instituciones y la influencia en el poco interés de los líderes de las naciones latinoamericanas en aportar en temas que involucran la investigación. Para lograr esto se debe aportar en la construcción de instituciones de la ciencia y el desarrollo tecnológico e incentivar a todas aquellas personas que se inclinan en brindar nuevas ideas y soluciones a través de la investigación de temas nuevos y de la comprobación en temas ya desarrollados.

En el estudio, se observó que durante años, los países de Latino América han incrementado la producción en la investigación científica a pesar de las limitaciones. Sin embargo, sus porcentajes aún se mantienen bajos en comparación a los países desarrollados, el incremento se debe al interés de personas que se inclinan en desarrollar proyectos de ciencias para ser reconocidos a nivel internacional y que a pesar de las restricciones económicas buscan ayuda por medios internacionales dando a conocer sus resultados en proyectos de investigación.

Palabras clave: Evolución, investigación, ciencia, Latinoamérica. 


\title{
Evolución de la Investigación Científica en América Latina
}

Vol. 2, núm. 2., (2018)

Katuska Isabel Cepeda Ávila; Lucila Pazmiño Iturralde; Eva Leonor Medrano Freire

\begin{abstract}
This work synthesizes the evolution in scientific research in Latin America through the years and everything related to the growth in the production of research in science and technology of the word to scientific publications at the international level. The development of scientific research on the part of institutions and the influence on the interest of the leaders of Latin American nations in contributing to the issues that involve research. To achieve this, we must contribute to the construction of science and technology development institutions and encourage all those who are inclined to offer new ideas and solutions through the investigation of new topics and security issues.
\end{abstract}

In the study, that was done for years, the countries of Latin America have increased production in scientific research despite the limitations. However, their percentages are still low compared to the components, the increase is due to the interest of people who are inclined in the development of projects for sciences for the international level and that despite the economic limitations they seek help through the international media, making its results known in research projects.

Key words: Evolution, research, science, latin america. 


\section{Evolución de la Investigación Científica en América Latina}

Vol. 2, núm. 2., (2018)

Katuska Isabel Cepeda Ávila; Lucila Pazmiño Iturralde; Eva Leonor Medrano Freire

\section{Introducción.}

El termino investigación engloba un estudio orientado al descubrimiento por medio de nuevos conocimientos y su aplicación en la solución de problemas e interrogantes a través de la recopilación de datos, información y cualquier hecho que se relacione a algún tema en específico.

En la actualidad el desarrollo de la investigación se presenta a diario, los seres humanos están todo el tiempo innovando y en la búsqueda de cosas modernas para el mundo y la ciencia. Existe una especie de sed de saber, aprender, descubrir, innovar y comprobar todo a su paso lo que incentiva el desarrollo de la investigación en toda clase de ámbitos.

Sin embargo, (Significados.com, 2016) define,

La investigación científica como un proceso ordenado y sistemático de indagación en el cual, mediante la aplicación rigurosa de un conjunto de métodos y criterios, se persigue el estudio, análisis o indagación en torno a un asunto o tema, con el objetivo subsecuente de aumentar, ampliar o desarrollar el conocimiento que se tiene de este.

"La investigación científica es un proceso libre y creativo, esto no quiere decir que carezca de sistematicidad y organización, mucho menos si se trata de la etapa de planificación, la cual se concreta con el proyecto de investigación" (Arias, 1999, p. XI). 


\section{Evolución de la Investigación Científica en América Latina}

Vol. 2, núm. 2., (2018)

Katuska Isabel Cepeda Ávila; Lucila Pazmiño Iturralde; Eva Leonor Medrano Freire

A pesar de que la sociedad latinoamericana se ha acostumbrado a esperar que los nuevos avances científicos y tecnológicos vengan de los países desarrollados más que de sus propios científicos. (Andes, 2017), con los años se ha ido incrementando su desarrollo. Los proyectos de investigación son implementados en instituciones y universidades con el fin de encontrar solución a problemas que orientan a descubrir algo desconocido o a comprobar resultados de trabajos de investigación previos para aportar ideas, mejoras y nuevas posibles soluciones.

No obstante, aunque América Latina aumentan cada vez más el número de científicos e instituciones de investigación, la ventaja que hay por parte de los países desarrollados es muy grande. Los países Latinoamericanos no brindan una importancia primordial en el desarrollo de la ciencia y la tecnología. Esto se debe a que la asignación de recursos para la investigación está en gran desproporción respecto a los países desarrollados, por lo tanto, no es falta de creatividad por parte de sus científicos, sino más bien, por el desinterés existente en quienes lideran las naciones.

Los principales factores que contribuyen a la baja productividad científica son el acceso limitado a subsidios para investigación, presupuestos inadecuados, niveles deficientes de infraestructura de laboratorios de investigación y de sus equipamientos. (Andes, 2017). Los científicos latinoamericanos incursionan en las competencias de temas de interés internacional para optar por becas y promociones que los ayude en sus carreras científicas. 


\section{Evolución de la Investigación Científica en América Latina}

Vol. 2, núm. 2., (2018)

Katuska Isabel Cepeda Ávila; Lucila Pazmiño Iturralde; Eva Leonor Medrano Freire

Probablemente la ciencia no sea el motor primordial de la economía en los países de Latinoamérica, sin embargo cada día existen más jóvenes graduados que desean aportar sus conocimientos científicos a la nación e innovar en temas para el desarrollo de sus países. Esto debería ser un tema de gran importancia para todos los países latinoamericanos, priorizar el desarrollo científico y tecnológico brindando las herramientas necesarias a instituciones y científicos interesados en la investigación, ya que, esto impulsa el futuro de la nación.

\section{Método y materiales.}

Esta investigación está enfocada en el estudio de la evolución científica en América Latina y su incremento en el desarrollo de la investigación, a través de la recopilación de información importante que direccionan al conocimiento científico y al crecimiento de las publicaciones de los países latinoamericanos a nivel internacional. Por tanto, se trata de una revisión bibliográfica vigente sobre la incremento de la producción e investigación de la ciencia en las naciones Latinoamérica.

La revisión se ha centrado en textos, documentos y artículos científicos publicados disponibles en la web, considerando que aquella herencia de la globalización nos permite acceder a mayor y mejor información a través de las herramientas tecnológicas. El motor de búsqueda ha sido herramientas académicas de la web que direccionan específicamente a archivos con validez y reconocimiento científico, descartando toda información no confirmada o sin las respectivas referencias bibliográficas. 


\section{Evolución de la Investigación Científica en América Latina}

Vol. 2, núm. 2., (2018)

Katuska Isabel Cepeda Ávila; Lucila Pazmiño Iturralde; Eva Leonor Medrano Freire

\section{Resultados}

A pesar de que los avances científicos y tecnológicos tienen mayor impacto en los países desarrollados, con los años los países latinoamericanos han evolucionado en estos temas incrementando el desarrollo científico en universidades e instituciones, con el fin de formar individuos capaces de brindar solución a problemas y nuevas opciones en el desempeño de la investigación de diferentes temas de la ciencia.

En un estudio realizado por Albornoz, "El total de documentos científicos de difusión internacional publicados en los 24 países de América Latina y el Caribe durante el periodo 1999-2002, en temas de Ciencias Experimentales, Humanidades y Ciencias Sociales, ascendió a 96988. De ellos, el 93\% corresponde a los publicados en SCI (ciencias experimentales), alrededor del 4\% en SSCI (ciencias sociales) y un 3\% en A\&HCI (arte y humanidades). Teniendo en cuenta solamente los resultados de ciencias experimentales la cifra supone un aumento del 64\% respecto a lo publicado en el periodo 1991-1995”. (2009).

Los porcentajes fueron tomados de la base de datos documental de la Science Citation Index (SCI) para los documentos de ciencias experimentales, de Social Sciences Citation Index (SSCI) para los de ciencias sociales y de Arts \& Humanities Citation Index (A\&HCI) para los de arte y humanidades. El cual se denomina base de datos del Institute for Scientific Information (ISI) creado por Thomson Reuters cuando se refiere a las tres ramas en conjunto.

De acuerdo al estudio realizado en la base de datos de (SCI), el incremento de la investigación y publicación de documentos ha aumentado en un 140\% desde 1991 al 2002. Este 


\section{Evolución de la Investigación Científica en América Latina}

Vol. 2, núm. 2., (2018)

Katuska Isabel Cepeda Ávila; Lucila Pazmiño Iturralde; Eva Leonor Medrano Freire

desarrollo en la producción científica, conlleva a una mayor amplitud en el reconocimiento de la ciencia en América Latina para el mundo. (Albornoz, 2009).

Sin embargo, a pesar de los esfuerzos realizados por parte de los países como Brazil, Colombia, Argentina, Chile, Venezuela, entre otros, en la edición de documentos científicos, el porcentaje de publicación para revistas que son editadas en América Latina no equivale a un cifra significativa, lo que lleva a los científicos latinoamericanos a buscar la manera de realizar sus publicaciones en revistas fuera de la región para dar a conocer sus resultados en investigaciones científicas. Esto se debe al desinterés existente por parte de los países latinoamericanos en implantar políticas que impulsen aún más el desarrollo de la investigación, así como, la creación de equipos e instituciones con el aporte económico de la nación que fomenten la investigación de calidad.

Ríos \& Herrero (2005), plantean que la ciencia de los países latinoamericanos es publicada a través de revistas nacionales que están poco representadas en bases de datos internacionales. No obstante en estudios realizados se observa que la investigación científica ha venido evolucionando con el pasar de los años.

A continuación se presenta un gráfico en el que se observa la evolución de la investigación científica a nivel mundial 


\section{Evolución de la Investigación Científica en América Latina}

Vol. 2, núm. 2., (2018)

Katuska Isabel Cepeda Ávila; Lucila Pazmiño Iturralde; Eva Leonor Medrano Freire

Imagen $\mathrm{N}^{\circ} 1$. Evolución de la producción científica por regiones del mundo.

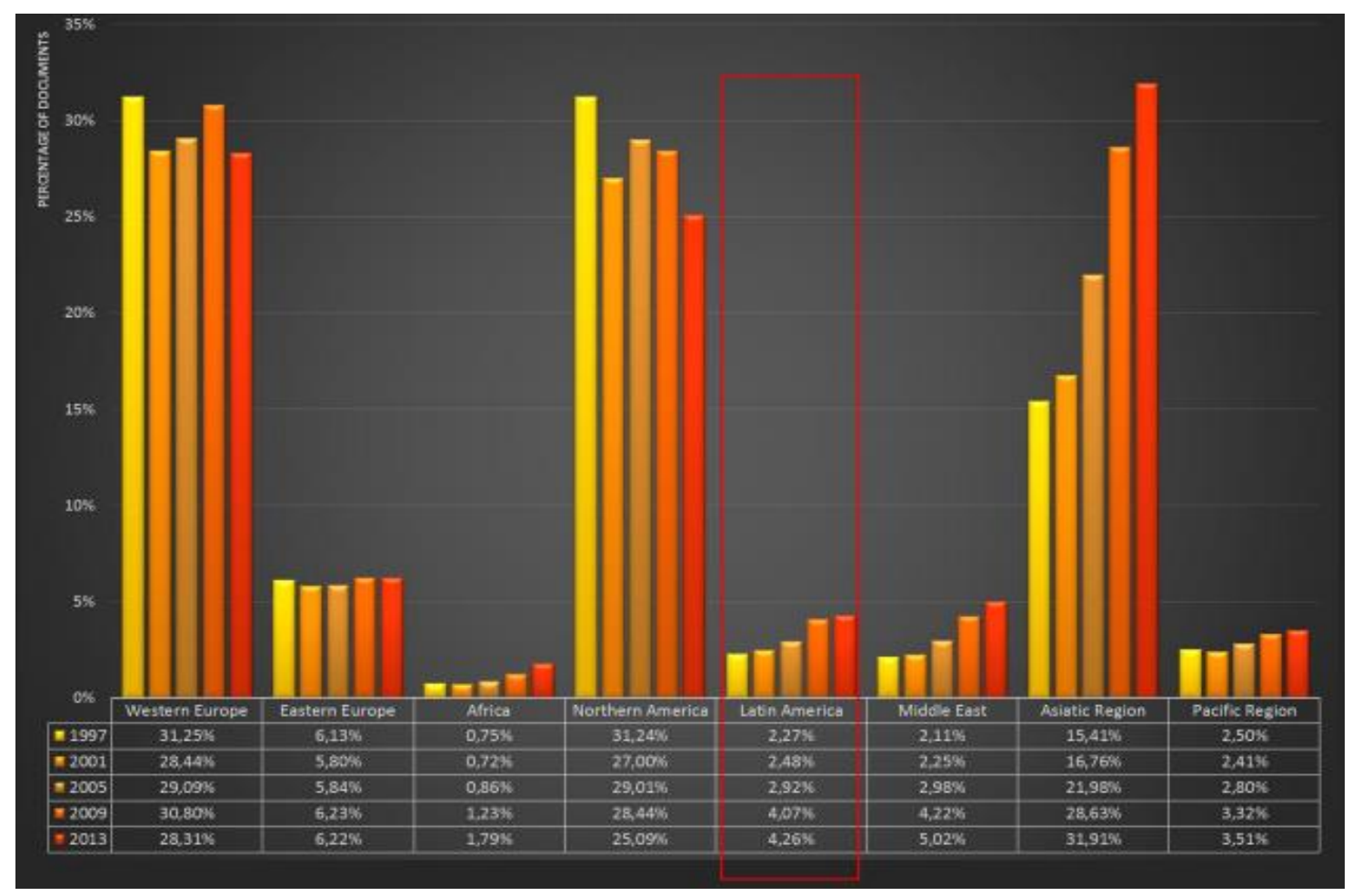

Fuente: (González, 2015).

En el grafico se observa que, los países de Europa Oriental así como los países de Norte América y la Región Asiática representan el mayor porcentaje en la producción científica a nivel mundial, a pesar de que el porcentaje para el año 1997 de la región Europa Oriental era mayor que para el año 2013 su rango de producción en promedio se mantiene, lo mismo se observa en la región de Norte América. A diferencia de la región Asiática donde se aprecia un incremento casi del doble en la producción científica desde 1997 hasta el 2013.

En el grafico también se observa que la región latinoamericana ha aumentado en casi el doble su porcentaje en las investigaciones científicas desde el 1997 al 2013. Esto producto de la 


\section{Evolución de la Investigación Científica en América Latina}

Vol. 2, núm. 2., (2018)

Katuska Isabel Cepeda Ávila; Lucila Pazmiño Iturralde; Eva Leonor Medrano Freire

creación de institutos y de la colaboración por parte de otros países interesados en los proyectos desarrollados por científicos latinoamericanos.

Sin embargo, a pesar de que la producción en las investigaciones científica para las regiones de Europa Oriental, Norte América y Asia es mayor, el promedio de crecimiento de producción científico es bajo. A continuación se presenta un gráfico en donde se explica lo anteriormente expuesto:

\section{Imagen $N^{\circ}$. Crecimiento promedio de la producción científica de las regiones del mundo}

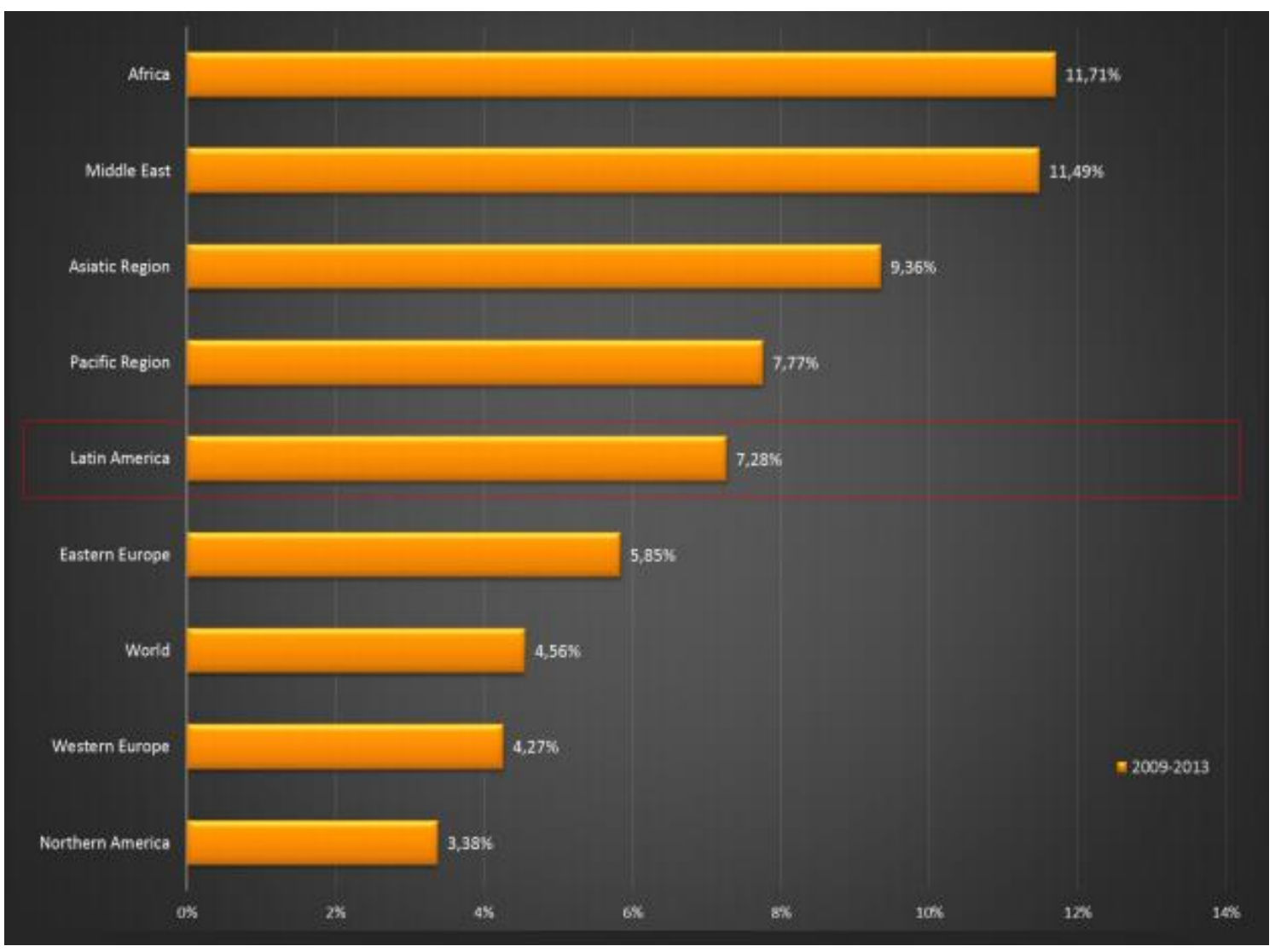

Fuente: (González, 2015) 


\section{Evolución de la Investigación Científica en América Latina}

Vol. 2, núm. 2., (2018)

Katuska Isabel Cepeda Ávila; Lucila Pazmiño Iturralde; Eva Leonor Medrano Freire

En la gráfica se observa claramente que aquellas regiones donde la producción científica es elevado su crecimiento desde 1997 hasta el año 2013 es relativamente bajo, sin embargo los países donde la producción científica es menor se observa un porcentaje mayor en el crecimiento o desarrollo científico, esto se debe al esmero por parte de las regiones de poca producción en aumentar su crecimiento y competir entre las grandes potencias incentivando a aquellos que se dedican a la investigación.

Por tanto, en un análisis de los resultados desde años anteriores hasta casi la actualidad, la investigación científica en América Latina ha incrementado su crecimiento a pesar de las limitaciones que existen por parte del desinterés en los lideres de cada nación en aportar las herramientas económicas necesarias y de aquella costumbre de esperar que los nuevos avances científicos y tecnológicos vengan por parte de los países desarrollados.

\section{Discusión y Conclusiones}

En el estudio de la evolución de la investigación científica en América Latina se ha observado que por medio de la revisión e investigación dentro de un rango de años considerables el desarrollo de la investigación científica y la publicación de sus resultados se han incrementado considerablemente.

Durante la década de los 90 y principios de los 2000 se ve un gran auge en la producción y desarrollo de la investigación científica (Ríos Gómez, 2005), sin embargo para los años subsiguientes se observa que a pesar de que los porcentajes son bajos respecto a los países desarrollados, América Latina ha incrementado sus temas de investigación científica. 


\section{Evolución de la Investigación Científica en América Latina}

Vol. 2, núm. 2., (2018)

Katuska Isabel Cepeda Ávila; Lucila Pazmiño Iturralde; Eva Leonor Medrano Freire

A pesar de que los países con menos producción científicos se ven influenciados por el poco aporte por parte de los líderes de la nación, sus científicos hacen sus mayores esfuerzos en darse a conocer a nivel internacional o percibir alguna ayuda que costee los gastos que acarrea una investigación científica, lo que indica que los investigadores latinoamericanos se han derivado hacia revistas de fuera de la región para publicar sus resultados científicos y ser reconocidos a nivel mundial.

Una opción podría ser, la construcción de más instituciones que aportan al desarrollo científico en los diferentes países de Latinoamérica, que incentive el interés en investigar, descubrir, y aportar nuevas soluciones y conocimientos a problemas en las diferentes ramas de la ciencia.

Seria de suma importancia que los líderes de cada región Latinoamericana centraran su importancia en el impulso del desarrollo de la investigación en todas las áreas existente y de tal manera explotar este potencial en la educación de cada uno de sus habitantes con el fin de aportar el desarrollo de la nación.

Finalmente se puede concluir que, aunque la evolución científica en Latinoamérica ha aumentado con el paso de los años, el incremento por parte de esta región del mundo sería mayor si las naciones prestaran más interés en el tema de la ciencia y la tecnología ayudando a quienes se interesan por crear nuevos aportes y realizar investigaciones que impulsan al desarrollo global.

\section{Bibliografía.}

Albornoz, M. ( 2009). Indicadores de innovación: las dificultades de un concepto en evolución. Revista iberoamericana de ciencia tecnología y sociedad, 5(13), 9-25. 


\section{Evolución de la Investigación Científica en América Latina}

Vol. 2, núm. 2., (2018)

Katuska Isabel Cepeda Ávila; Lucila Pazmiño Iturralde; Eva Leonor Medrano Freire

Andes, L. (2017). Obtenido de https://losandes.com.ar/article/investigacion-cientifica-enamerica-latina-una-evaluacion-desde-adentro

Arias, F. G. (1999). El proyecto de investigación. Fidias G. Arias Odón.

Claudia Ríos Gómez, V. H. (2005). LA PRODUCCIÓN CIENTÍFICA LATINOAMERICANA Y LA CIENCIA MUNDIAL: UNA REVISIÓN BIBLIOGRÁFICA (1989-2003).

González, D. A. (2015). La producción científica de America Latina en el escenario mundial.

Ríos Gómez, C. \&. (2005). La producción científica latinoamericana y la ciencia mundial: una revisión bibliográfica (1989-2003). Revista interamericana de Bibliotecología, 28(1), 4361.

Significados.com. (2016). Obtenido de https://www.significados.com/investigacion-cientifica/ 